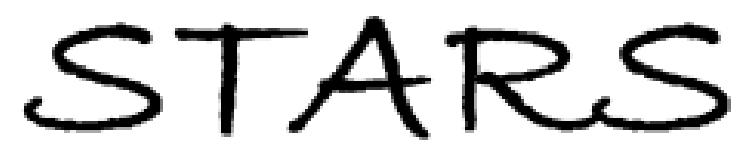

University of Central Florida

STARS

$1-1-1990$

\title{
Effects Of Using The More Accurate Intrinsic Concentration On Bipolar-Transistor Modeling
}

J. J. Liou

University of Central Florida

J. S. Yuan

University of Central Florida

W. W. Wong

University of Central Florida

Find similar works at: https://stars.library.ucf.edu/facultybib1990

University of Central Florida Libraries http://library.ucf.edu

This Article is brought to you for free and open access by the Faculty Bibliography at STARS. It has been accepted for inclusion in Faculty Bibliography 1990s by an authorized administrator of STARS. For more information, please contact STARS@ucf.edu.

\section{Recommended Citation}

Liou, J. J.; Yuan, J. S.; and Wong, W. W., "Effects Of Using The More Accurate Intrinsic Concentration On Bipolar-Transistor Modeling" (1990). Faculty Bibliography 1990s. 99.

https://stars.library.ucf.edu/facultybib1990/99

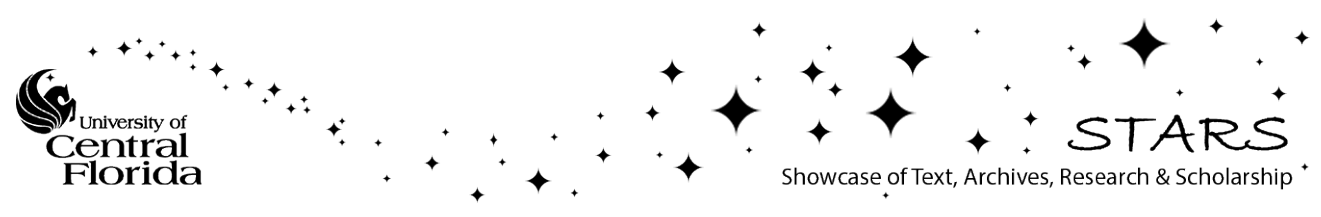




\section{Effects of using the more accurate intrinsic concentration on bipolar transistor modeling}

Cite as: Journal of Applied Physics 68, 5911 (1990); https://doi.org/10.1063/1.346942

Submitted: 09 April 1990. Accepted: 20 August 1990 . Published Online: 04 June 1998

J. J. Liou, J. S. Yuan, and W. W. Wong

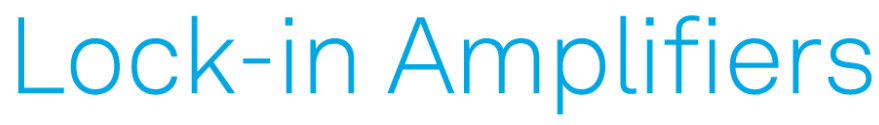

... and more, from DC to $600 \mathrm{MHz}$

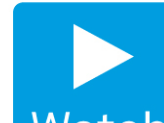

Watch 


\title{
Effects of using the more accurate intrinsic concentration on bipolar transistor modeling
}

\author{
J.J. Liou, J. S. Yuan, and W. W. Wong \\ Electrical Engineering Department, University of Central Florida, Orlondo, Florida 32816
}

(Received 9 April 1990; accepted for publication 20 August 1990)

\begin{abstract}
A more accurate intrinsic concentration was suggested recently. Discrepancies between using the conventional and the more accurate intrinsic concentrations on bipolar transistor modeling are assessed in this study. Our calculations show that the conventional intrinsic concentration overestimates the collector and base currents by a factor of 1.5 to 2 but affects less severely the steady-state current gain.
\end{abstract}

The intrinsic carrier concentration is an important parameter for semiconductor device modeling. ${ }^{1,2}$ The values of intrinsic concentration, effective densities of states, and effective mass in silicon have recently been reassessed theoretically as well as experimentally. ${ }^{3}$ It is found that the commonly used values are inconsistent. For example, for the silicon intrinsic concentration at room temperature, the critical evaluation results in a value of $1.08 \times 10^{10} \mathrm{~cm}^{-3}, 3$ instead of $1.45 \times 10^{10} \mathrm{~cm}^{-3}$ as conventionally used.

This study examines the effects of using the more accurate intrinsic concentration, as compared to using the conventional value, on modeling bipolar junction transistors (BITs).

Consider an $n / p / n$ BJT (Fig. 1) at room temperature uncer forward-active operation. Using the assumptions that the charge transport in the base is predominantly due to difusion and that the transistor has a thin base, the collector current density $J_{\mathrm{c}}$ can be expressed as

$$
\begin{aligned}
J_{c} & \approx q D_{n}\left[\Delta n\left(X_{2}\right)-\Delta n\left(X_{3}\right)\right] /\left(X_{3}-X_{2}\right) \\
& \approx q D_{n} \Delta n\left(X_{2}\right) /\left(X_{3}-X_{2}\right),
\end{aligned}
$$

where $D_{n}$ is the electron diffusion coefficient, $\Delta n$ is the excess electron concentration, and $X_{2}$ and $X_{3}$ are the edges of the space-charge layers defned in Fig. 1. Because the doping densities are highly asymmetrical in the emitter, base, and collector, we can assume one-sided junctions:

$X_{3}-X_{2} \approx X_{j c}-X_{j E}-\left[(2 \epsilon / q)\left(V_{\mathrm{biBE}}-V_{\mathrm{BE}}\right) / N_{B}\right]^{0.5}$

where $X_{j c}$ and $X_{j E}$ are the metallurgical junctions (Fig. 1), $\epsilon$ is the dielectric permittivity, $V_{\mathrm{HE}}$ is the base-emitter applied voltage, $N_{H}$ is the average base doping density and $V_{\text {hiBr }}$ is the emitter-base junction builtin potential. $\Delta n\left(X_{2}\right)$ in Eq. (1) for all injection levels is ${ }^{4}$

$$
\begin{aligned}
\Delta n\left(X_{2}\right)= & -0.5 N_{B} \\
& +0.5 N_{B}\left\{1+\left[4 n_{i}^{2} \exp \left(V_{B B} / V_{T}\right) / N_{B}^{2}\right]\right\}^{0.5},
\end{aligned}
$$

where $n_{i}$ is the intrinsic concentration and $V_{T}$ is the thermal voltage.

We next proceed to find the base current density $J_{B}$. For a thin-base bipolar transistor, $J_{B}$ is the sum of the recombination current density $I_{\mathrm{SCR}}$ in the emitter-base space-charge layer and the hole current density $J_{\mathrm{RF}}$ injected from the base to emitter. Thus ${ }^{5,6}$

$$
\begin{aligned}
J_{B}=J_{\mathrm{SCR}}+J_{\mathrm{RE}}= & 1.25\left(q V_{T} n_{i} / \tau\right)\left\{\left(q N_{E} / \epsilon\right)\right. \\
& \left.\times\left[2 V_{T} \ln \left(N_{E} / n_{i}\right)-V_{\mathrm{BE}}\right]\right\} \cdot 0.5 \\
& \times \exp \left(V_{\mathrm{BE}} / 2 V_{T}\right)+q D_{p} \Delta p\left(X_{1}\right) / X_{j E} .
\end{aligned}
$$

$\tau$ is the iffetime associated with the space-charge layer tecombination, $N_{E}$ is the average emitter doping concentration, $D_{p}$ is the hole diffusion coefficient, and $\Delta p$ is the excess hole density. For all injection levels,

$$
\begin{aligned}
\Delta p\left(X_{1}\right)= & -0.5 N_{H} \\
& +0.5 N_{E}\left\{1+\left[4 n_{i}^{2} \exp \left(V_{\mathrm{HE}} / V_{T}\right) / N_{E E}^{2}\right]\right\}^{0.5} .
\end{aligned}
$$

Note that $J_{\mathrm{SCR}}$ is described based on the simplest and widely used Shockley-Read-Hall model ${ }^{\top}$ which assumes that there is only one type of trap that dominates the recombination process and that the traps are uniformly distributed in the middle of band gap.

The common-emitter de current gain $\beta$ of the transistor is

$$
\beta=J_{c} / J_{B} \text {. }
$$

It is apparent from the foregoing analysis that the value of $n_{i}$ plays an important role in the BJT modeling. We first discuss qualitatively and intuitively the effect of using the

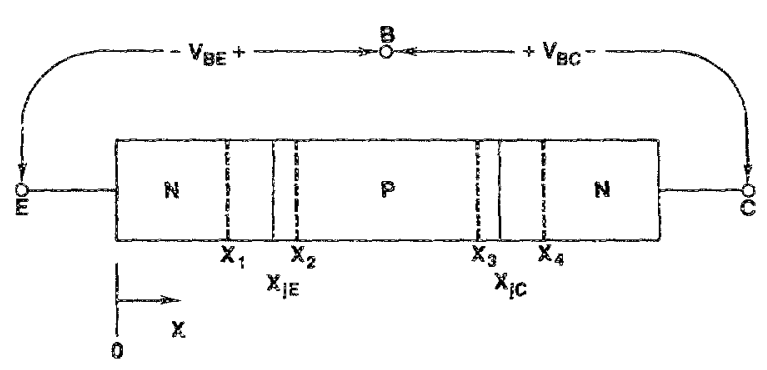

FIG. 1. One-dimensional bipolar transistor structure, where $X_{1}$ and $X_{2}$ are the edges of the emitter-base space-chatge layer and $X_{3}$ and $X_{4}$ are the edges of the base-collector space-charge layer. 


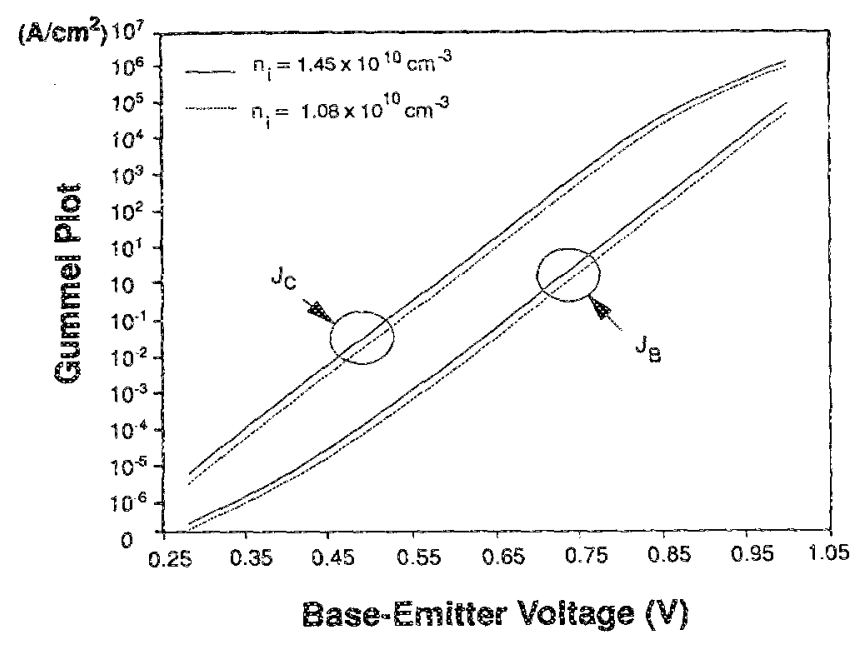

FIG. 2. Base and collector current densities calculated using the conventional intrinsic concentration and using the more accurate intrinsic concentration.

more accurate $n_{i}$ on $J_{c}, J_{B}$, and $B$. An exact number example will be given latter.

The current-voltage characteristics of a $\mathrm{BIT}$ can in general be divided into the following three regions: low bias region, medium-bias region, and high-bias region. From a fundamental viewpoint, the collector current is proportional to $\Delta n\left(X_{2}\right)$ which is a function of $n_{i}^{2}$ for low-and medium-bias conditions (low injection) and is a function of $n_{i}$ for high voltage (high injection). On the other hand, because $J_{\mathrm{SCR}} \propto \exp \left(V_{\mathrm{BE}} / 2 V_{T}\right)$ and $J_{\mathrm{RE}} \propto \exp \left(V_{\mathrm{BF}} / V_{T}\right)$, the base current is dominated by $J_{\mathrm{SCR}}$ (depends on $n_{j}$ ) at a low-bias condition and is dominated by $J_{\mathrm{RE}}$ (depends on $n_{i}^{2}$ if low injection prevails) at medium- and high-bias conditions. It should be pointed out that a low-injection condition can be assumed in the emitter because of the high doping concentration. Thus, $J_{C} \propto n_{i}^{2}, J_{B} \propto n_{i}$, and $\beta \propto n_{i}$ for a low-bias region, $J_{C} \propto n_{i}^{2}, J_{B} \propto n_{i}^{2}$, and $\beta$ becomes constant with respect to $n_{i}$ for a medium-bias region, and $J_{c} \propto n_{i}, J_{B} \propto n_{i}^{2}$, and $\beta \propto n_{i}^{-1}$ for a high-bias region. As a consequence, if the more accurate $n_{i}$ proposed by Green $\left(1.08 \times 10^{10} \mathrm{~cm}^{-3}\right)$ instead of the conventional $n_{i}\left(1.45 \times 10^{10} \mathrm{~cm}^{-3}\right)$ is used, $J_{C}$ will decrease by a factor of 0.55 in low-and medium-bias regions and decrease by a factor of 0.74 in a high-bias region, $J_{k}$ will decrease by a factor of 0.74 in a low-bias region and decrease by a factor of 0.55 otherwise, and $\beta$ will decrease by a factor of 0.74 in a low-bias region, remain the same in a mediumbias condition, and increase by a factor of 0.74 in a high-bias region.

To verify the above physical intuitions, we now calculate $J_{C}, J_{B}$, and $\beta$ using the conventional $n_{i}$ and the nore accurate $n_{i}$ for a typical advanced BJT having $N_{E}=10^{19}$ $\mathrm{cm}^{-3}, N_{B}=2 \times 10^{17} \mathrm{~cm}^{-3}, N_{C}=10^{16} \mathrm{~cm}^{-3}, X_{j E}=0.15$ $\mu \mathrm{m}$, and $X_{j C}=0.35 \mu \mathrm{m}$. Values for $D_{n}$ and $D_{p}$ used for cal. culations are the same as that suggested in $\operatorname{Ref} 3\left(D_{n}=36.8\right.$ $\mathrm{cm}^{2} / \mathrm{s}$ and $\left.D_{p}=12.2 \mathrm{~cm}^{2} / \mathrm{s}\right)$. Shown in Fig. 2 are the base and collector current densities versus the base-emitter ap-

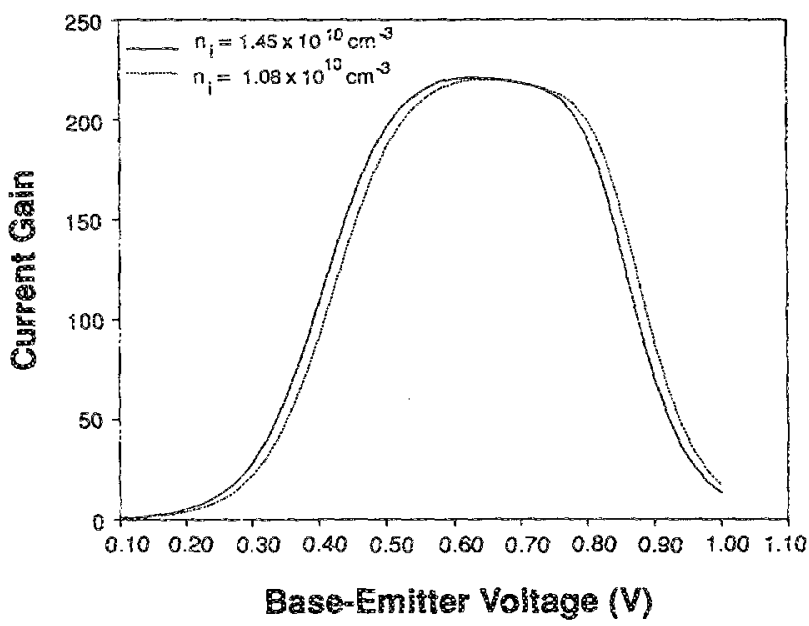

FIG. 3. Common-emitter current gain calculated using the conventional. intrinsic concentration and using the more accurate concentration

plied voltage (Gummel plot) using the more accurate $n_{i}$ and the conventional $n_{i}$. Current gain versus voltage characteristics are given in Fig. 3. The results in Fig. 2 confirm our earlier discussions and the postulate by Green that the use of the conventional $n$, value overestimates the current flow in silicon devices. ${ }^{3}$ Specifically, the conventional $n_{i}$ overestimates the collector current and the base current by a factor of 1.5 to 2 depending on the bias condition. However, as shown in Fig. 3, the errors in modeling the current gain originated from the conventional $n_{i}$ is less severe (by a factor of 1.3 or less) because the two current overestimations counterbalance each other. Note that the trend in Fig. 3 agrees well with our qualitative prediction; using the more accurate $n_{i}$ results in a smaller $\beta$ in a low-bias region, about the same $\beta$ in a medium-bias region, and a larger $\beta$ in a high-bias region.

In conclusion, the intrinsic concentration was recently reassessed experimentally and theoretically. We have examined the effects of using this more accurate intrinsic concentration on the bipolar trausistor modeling at room temperature. It is found that the use of the conventional intrinsic concentration results in overestimating both the collector and base currents by a factor of 1.5 to 2 . On the other hand, the dc current gain, which is a critical parameter for the bipolar transistor performance, is less sensitive to the difference between the two intrinsic concentrations.

'S. M. Sze, Physics of Semiconductor Devices, 2nd ed. (Wiley, New York, $1981)$.

2E. S. Yang, Microelectronics Devices (McGraw-Hill, New York, 1988).

${ }^{3}$ M. A. Green, J. Appl. Phys. 67, 2944 (1990).

${ }^{4}$ R. S. Muller and T. 1. Kamins, Device Electronics for Integrated Circuits 2nd ed. (Wiley, New York, 1986).

${ }^{5}$ M. Shur, Physics of Semiconductor Devices (Prentice Hall, Englewood Cliffs, N3, 1990).

${ }^{6} \mathrm{M}$. Shur, IEEE Trans. Electron Devices 35, 1564 (1988).

${ }^{7}$ W. Shockley and W. T. Read, Phys. Rev. 87,835 (1952). 